\title{
A NOTE ON PERIODIC SOLUTIONS FOR DELAY-DIFFERENTIAL SYSTEMS ${ }^{1}$
}

\author{
G. B. GUSTAFSON AND K. SCHMITT
}

ABstRACT. Let $f(t, x, y):[0, \infty) \times \boldsymbol{R}^{n} \times \boldsymbol{R}^{n} \rightarrow \boldsymbol{R}^{n}$ be continuous and 1-periodic in $t, \tau(t):[0, \infty) \rightarrow[0, h](0<h \leqq 1)$ continuous and 1-periodic. A simple geometric condition (Theorem 1) is given for the existence of 1-periodic solutions $x(t)$ of the nonlinear delaydifferential system $x^{\prime}(t)=f(t, x(t), x(t-\tau(t)))$, with $x(t)$ in a given bounded convex open set $G$ in $\boldsymbol{R}^{n}$. The addition of a Lipschitz condition in $x$ and monotonicity in $y$ allows one to calculate $x(t)$ by a monotone sequence of successive approximations (Theorem 2 ). Extensions to a more general functional differential equation $x^{\prime}(t)=g\left(t, x(t), x_{t}\right)$ are given.

1. Introduction. Let $f:[0, \infty) \times \boldsymbol{R}^{n} \times \boldsymbol{R}^{n} \rightarrow \boldsymbol{R}^{n}, \tau:[0, \infty) \rightarrow[0, h]$ be continuous, 1-periodic in the variable $t$, and consider the first order nonlinear differential system

$$
x^{\prime}(t)=f(t, x(t), x(t-\tau(t))) \quad\left({ }^{\prime}=d / d t\right) .
$$

The purpose of this note is to extend the range of applicability, and remove complicated hypotheses, in certain results of Mikolajska [3] concerning the existence and successive approximation of periodic solutions of (1) with $\tau(t) \equiv h \equiv 1$. We consider separately the question of successive approximations $(\$ 4)$; the existence is established by the following

THEOREM 1. Let $G$ be a bounded convex open set in $\boldsymbol{R}^{n}$ containing 0 , and and assume there is a function $N: \partial G \rightarrow R^{n}-\{0\}$ satisfying

$$
\begin{gathered}
N(x) \cdot x>0 \text { for } x \in \partial G, \\
G \subseteq\{y: N(x) \cdot(y-x) \leqq 0\} \text { for each } x \in \partial G .
\end{gathered}
$$

Suppose $f:[0, \infty) \times \boldsymbol{R}^{n} \times \boldsymbol{R}^{n} \rightarrow \boldsymbol{R}^{n}, \tau:[0, \infty) \rightarrow[0, h]$ are continuous functions, 1-periodic in the variable $t$ and

(4) $N(x) \cdot f(t, x, y)$ is positive (negative) for all $x \in \partial G, y \in G, t \in[0,1]$.

Then equation (1) has a 1-periodic solution $x(t)$ with values in $G$.

Received by the editors February 12, 1973.

AMS (MOS) subject classifications (1970). Primary 34J05; Secondary 34K10.

Key words and phrases. Periodic solutions, delay equations.

1 Research supported by the U.S. Army Research Office under Grant ARO-D-31124-72-G46.

(c) American Mathematical Society 1974 
CoRollaRy. Let $f:[0, \infty) \times \boldsymbol{R}^{n} \times \boldsymbol{R}^{n}, \tau:[0, \infty) \rightarrow[0, h]$ be continuous, and 1-periodic in the variable $t(0<h \leqq 1)$. Assume that there exists a number $R>0$ such that

$$
x \cdot f(t, x, y)>0 \text { for all }|x|=R,|y| \leqq R, t \in[0,1],
$$

or

$$
x \cdot f(t, x, y)<0 \text { for all }|x|=R,|y| \leqq R, t \in[0,1] .
$$

Then equation (1) has a 1-periodic solution with values in $|x|<R$. (In the sequel, $|\cdot|$ denotes the Euclidean norm in $R^{n}$, and $x \cdot y$ is the usual inner product.)

REMARK. As noted in the corollary, it is often convenient to choose the set $G$ to be a sphere with center 0 . For second order scalar equations, it is sometimes more natural for $G$ to be a rectangle with barycenter 0 , and one can easily construct examples where a triangle leads to the simplest calculations.

We remark that $N(x)$ is not required to be continuous (see Theorem 2 for an application).

Conditions (2), (3) simply say that $N(x)$ is an outer normal for $G$, while relation (5) says that the outer normal $N(x)$ and the field $f$ are in the same (or opposite) half-space determined by the hyperplane $N(x) \cdot[y-x]=0$.

The above theorem was motivated by results of $Z$. Mikolajska [3], where functions similar to Lyapunov functions were used to establish the existence of periodic solutions of a scalar equation (1), with $\tau(t) \equiv h$, and period 1 replaced by period $h$. In honesty, these equations are really not delay equations from the point of view of periodic solutions, because the delay can be transformed out of the problem by a change of variables.

In this note, we obtain a number of improvements on the existence results in [3]. First, we consider equations (1) that are truly delay equations, and point out how to extend the results to more general functional differential equations (see $\S 3$ ). Secondly, we note that the complicated conditions B and B* of Mikolajska [3] imply the simple condition (5) of the corollary above, hence existence in [3] follows from our theorem. Finally, our theorem gives a simple geometric condition for existence of periodic solutions of systems (rather than scalar equations), without assuming monotonicity of $f$, or that $f$ is Lipschitzian. In addition, our result is obtained by a very short proof, in which we appeal to Brouwer degree calculations, and an elegant theorem of V. V. Strygin [5].

2. Proof of Theorem 1. Let us show that our theorem follows as a simple application of a theorem of V. V. Strygin [5]. In order to apply the result in [5], we first define a continuum of nested convex regions $G_{\lambda}$, $\lambda \geqq 0$, as follows. Given $x \notin G$, let $\bar{x}$ be the positive constant multiple of $x$ 
on $\partial G$, and put

$$
\begin{aligned}
& G_{\lambda}=\{x \notin G:|x-\bar{x}|<\lambda|x|\} \cup G, \quad \lambda>0, \\
& G_{0} \equiv G .
\end{aligned}
$$

The function $f(t, x, y)$ is modified, for purposes of the proof, as follows. Let $f_{1}$ be defined by

$$
\begin{aligned}
f_{1}(t, x, y) & =f(t, x, y), & & x \in \bar{G}, \\
& =f(t, \bar{x}, y), & & x \notin \bar{G} .
\end{aligned}
$$

Then define the modification $F_{\varepsilon}$ of the function $f$ by the formula

$$
\begin{aligned}
f_{\varepsilon}(t, x, y) & =\varepsilon f_{1}(t, x, y), & & y \in \bar{G}, \\
& =\varepsilon f_{1}(t, x, \bar{y}), & & y \notin \bar{G} .
\end{aligned}
$$

We observe that $F_{\varepsilon}$ is continuous in all its variables. The idea is to show that the modified equation

$$
x^{\prime}(t)=F_{\varepsilon}(t, x(t), x(t-\tau(t))) \quad(0<\varepsilon \leqq 1)
$$

has a 1-periodic solution $x(t)$ in $G$ for $\varepsilon=1$. It then follows that $x(t)$ is a 1 -periodic solution of equation (1) with values in $G$.

It will be shown that all possible 1-periodic solutions of equation (*) have values in $G$. Let $x(t)$ be a 1-periodic solution of (*), and assume, in order to obtain a contradiction, that $x(t)$ belongs to $\partial G_{\lambda}$ for some $\lambda \geqq 0$. Then there exists a maximal $\lambda \geqq 0$ for which this is true. Hence, there is a point $t_{0}$ with $x\left(t_{0}\right) \in \partial G_{\lambda}$, and $x(t) \in G_{\lambda}$ for all other values of $t$. In particular, $x(t-\tau(t)) \in G_{\lambda}$, so we may apply condition (4) to obtain for $x_{0}=$ $x\left(t_{0}\right), y_{0}=x\left(t_{0}-\tau\left(t_{0}\right)\right)$, the relation (assume the positive sign in (4); the reasoning is similar for the negative sign)

$$
N\left(\bar{x}_{0}\right) \cdot F_{\varepsilon}\left(t_{0}, x_{0}, y_{0}\right)>0 \text {. }
$$

Let us write $x\left(t_{0}+h\right)=x\left(t_{0}\right)+\int_{0}^{1} x^{\prime}\left(t_{0}+s h\right) h d s$, and choose $h>0$ so small that $N\left(\bar{x}_{0}\right) \cdot F_{\varepsilon}(t, x(t), x(t-\tau(t)))>0$ for $t_{0} \leqq t<t_{0}+h$. Then equation (*) gives

$$
N\left(\bar{x}_{0}\right) \cdot\left[x\left(t_{0}+h\right)-x\left(t_{0}\right)\right]>0 .
$$

Since $z_{0} \equiv x\left(t_{0}+h\right) \in G_{\lambda}$, and $x_{0} \in G_{\lambda}$, it follows that

$$
\mu=\left|z_{0}\right|^{-1}\left|z_{0}-\bar{z}_{0}\right| \leqq \lambda=\left|x_{0}\right|^{-1}\left|x_{0}-\bar{x}_{0}\right|,
$$

or else $z_{0} \in G$. The case $z_{0} \in G$ is eliminated by relations (**) and (3). 
Indeed, $x_{0}=a \bar{x}_{0}, a \geqq 1$, gives

$$
0<N\left(\bar{x}_{0}\right) \cdot\left[z_{0}-x_{0}\right]=a N\left(\bar{x}_{0}\right) \cdot\left[a^{-1} z_{0}-\bar{x}_{0}\right],
$$

but $a^{-1} z_{0} \in G$ by convexity, so (3) gives the reverse sign in this inequality. On the other hand, if $z_{0} \notin G$, then $z_{0}=b \bar{z}_{0}, b>1$. We see that $\mu=1-b^{-1}$, $\lambda=1-a^{-1}$, and therefore $\mu \leqq \lambda$ gives $b \leqq a$. But then

$$
0<N\left(\bar{x}_{0}\right) \cdot\left[z_{0}-x_{0}\right]=a N\left(\bar{x}_{0}\right) \cdot\left[a^{-1} b \bar{z}_{0}-\bar{x}_{0}\right] .
$$

However, $a^{-1} b \leqq 1$ implies $a^{-1} b \bar{z}_{0} \in G$, and by (3) the sign must be reversed. Therefore, in both cases, a contradiction is reached. This proves that all possible 1-periodic solutions of equation (*) have values in $G$.

Let us calculate the Brouwer degree $d(T, G, 0)$ of the mapping

$$
T x=\int_{0}^{1} F_{\varepsilon}(t, x, x) d t, \quad x \in G, \quad 0<\varepsilon \leqq 1 .
$$

First, $F_{\varepsilon}(t, x, x)=\varepsilon f(t, x, x)$ for $x \in G$; therefore (if (4) is positive)

$$
N(x) \cdot T x>0, \quad x \in \partial G .
$$

Secondly, we may apply the Poincaré-Bohl transformation

$$
H(x, \lambda)=\lambda T x+(1-\lambda) x, \quad x \in G, \quad 0 \leqq \lambda \leqq 1,
$$

to the identity and $T$, observe that $N(x) \cdot H(x, t)>0$ on $\partial G, 0 \leqq t \leqq 1$, because of (2) and (4), and conclude, by invariance of Brouwer degree under homotopy [4], that the degree in question is unity, hence nonzero.

We may now apply the result of V. V. Strygin $[5$, p. 601] to equation (*) and obtain a 1-periodic solution $x(t)$ of the modified equation with $x(t) \in G, 0 \leqq t \leqq 1$, for $\varepsilon=1$. The definition of the modified equation shows that $x(t)$ is a 1-periodic solution of equation (1), and the proof is complete.

3. Generalizations. Instead of equation (1), we may also consider the more general functional equation

$$
x^{\prime}(t)=f\left(t, x(t), x_{t}\right),
$$

where $f:[0, \infty) \times R^{n} \times C\left([-h, 0] \rightarrow R^{n}\right) \rightarrow R^{n}$ is continuous, and $x_{t} \in$ $C\left([-h, 0] \rightarrow R^{n}\right)$ is given as usual by the formula $x_{t}(\theta)=x(t+\theta),-h \leqq$ $\theta \leqq 0$. The very same theorem is valid for equation (6), provided we assume that $0<h \leqq 1, f$ is 1 -periodic in $t$, relations (2), (3) hold, and (4) is replaced by

$$
\begin{aligned}
& \text { For all } x \in \partial G, y \in C([-h, 0] \rightarrow G), t \in[0,1], \\
& \text { we have } N(x) \cdot f(t, x, y)>0 \text { (or negative). }
\end{aligned}
$$

The proof of $\$ 2$ extends to this setting with only minor modifications. 
4. Successive approximations. It is shown by Z. Mikolajska [3] that equation (1) with $\tau(t) \equiv h$ has a periodic solution $x(t)$ which is the limit of a decreasing sequence of successive approximations. To do this, Mikolajska imposes a complicated hypothesis (hypothesis B or B*) involving a function $V(t, x)$ similar to a Lyapunov function, and, in addition, it is assumed that $f(t, x, y)$ is increasing in $y, h$-periodic in $t$, and Lipschitz continuous in $x$. We replace Mikolajska's conditions $\mathrm{B}$ and $\mathrm{B}^{*}$ by a simple geometric condition, apply Theorem 1, and obtain the following result for systems, which is more tractable for special equations.

Theorem 2. Let $g:[0, \infty) \times \boldsymbol{R}^{n} \times \boldsymbol{R}^{n} \rightarrow \boldsymbol{R}^{n}, \tau:[0, \infty) \rightarrow[0, h](0<h \leqq 1)$ be continuous and 1-periodic in $t$, with $g(t, x, y)$ Lipschitz continuous in $x, g_{i}(t, x, y)$ is increasing with respect to $y_{k}, k \neq i, 1 \leqq k \leqq n, 1 \leqq i \leqq n$, and

(i) $x_{i} g_{i}(t, x, y)>0$ for $\left|x_{i}\right|=R, \max _{1 \leqq j \leqq n}\left|x_{j}\right| \leqq R, \max _{1 \leqq j \leqq n}\left|y_{j}\right| \leqq R$, $i=1,2, \cdots, n$, or

(i) the reverse inequality holds in (i).

Then there is a sequence $\left\{x^{n}(t)\right\}_{n=0}^{\infty}$ of 1-periodic functions satisfying

(ii) $-R<x_{i}^{n+1}(t)<x_{i}^{n}(t)<R, n \geqq 1,1 \leqq i \leqq n$,

(iii) $x_{i}^{0}(t) \equiv R, 1 \leqq i \leqq n$, and

$$
x_{n+1}^{\prime}(t)=g\left(t, x_{n+1}(t), x_{n}(t-\tau(t))\right), \quad n>0,
$$

which converge uniformly to a 1-periodic solution of

$$
x^{\prime}(t)=g(t, x(t), x(t-\tau(t))) .
$$

Proof. Let $x^{0}(t)$ be given by (iii), let $G$ be the parallelopiped defined by $\left|x_{i}\right|<R(1 \leqq i \leqq n)$. For $x \in \partial G$, let $N(x)=\left(\varepsilon_{1}, \cdots, \varepsilon_{n}\right)$ where $\varepsilon_{i}=0$ if $\left|x_{i}\right|<R, \varepsilon_{i}=x_{i}$ if $\left|x_{i}\right|=R, i=1, \cdots, n$. Then relations (2), (3) are easily verified. It is easy to see that (i) or (i)' implies (4), with $f$ replaced by $g$. Let us apply Theorem 1 to $f(t, x, y)=g\left(t, x, x^{0}(t)\right)$, where $x^{0}(t)$ is defined in (iii). Then there exists a periodic solution $x^{1}(t)$ of $x^{\prime}(t)=g\left(t ; x(t), x^{0}(t)\right)$ with values in $G$, and this gives inequality (ii) for $n=0$.

To establish the existence of the sequence $\left\{x^{n}(t)\right\}$, we proceed by induction, armed with techniques similar to those in Lemma 2, p. 28, in Mikolajska [3]. For this purpose, one needs a vector comparison theorem (see for example, P. Hartman [2, Example 4.1(6), p. 28]), in order to show that the set

$$
Z^{*}=\left\{x \in R^{n}:-R \leqq x_{i} \leqq x_{i}^{n}(0), 1 \leqq i \leqq n\right\}
$$

is mapped back into itself by the mapping

$$
T^{*}: x \rightarrow y(1, x), \quad y^{\prime}(t, x)=g\left(t, y(t, x), x^{n}(t)\right), \quad y(0, x)=x .
$$


The Lipschitz condition makes $T^{*}$ continuous, and the Brouwer fixed point theorem $[4, \mathrm{pp} .74,96]$ produces a fixed point of $T^{*}$, say $x_{0}$. Then $x^{n+1}(t)=y\left(t, x_{0}\right)$ is the desired periodic solution, completing the induction.

We use an Arzela-Ascoli theorem argument together with Dini's theorem to complete the proof.

COROLlaRY. Let $g:[0, \infty) \times \boldsymbol{R}^{1} \times \boldsymbol{R}^{1} \rightarrow \boldsymbol{R}^{1}, \tau:[0, \infty) \rightarrow[0, h](0<h<1)$ be continuous, 1-periodic in $t, g(t, x, y)$ Lipschitz continuous in $x$ and increasing in $y$, with

$$
g(t, R, y) g(t,-R, y)<0, \quad 0 \leqq t \leqq 1, \quad(y) \leqq R .
$$

Then there is a decreasing sequence of 1-periodic functions with values in $(-R, R)$ satisfying $x_{n+1}^{\prime}(t)=g\left(t, x_{n+1}(t), x_{n}(t)\right)$, with $\left\{x_{n}\right\}$ uniformly convergent to a 1-periodic solution of $x^{\prime}(t)=g(t, x(t), x(t-\tau(t)))$.

EXAMPLE. Let $f(t, y):[0, \infty) \times \boldsymbol{R}^{1} \rightarrow \boldsymbol{R}^{1}, a(t):[0, \infty) \rightarrow \boldsymbol{R}^{1}, \tau(t):[0, \infty) \rightarrow$ $[0, h](0<h \leqq 1)$ be continuous and 1-periodic in $t$, with $f$ increasing in $y$, $|a(t)|>0$, and

$$
\lim _{R \rightarrow \infty} R^{-1} \cdot \sup \{|f(t, y)|: 0 \leqq t \leqq 1,|y| \leqq R\}=0 .
$$

Then a periodic solution of $x^{\prime}(t)=a(t) x(t)+f(t, x(t-\tau(t)))$ can be computed by successive approximations as in the corollary.

REMARK. The theorem has an immediate extension to functional equations of the form $x^{\prime}(t)=g\left(t, x(t), x_{t}\right)$, which the reader can easily supply. The appropriate differential inequality theorem needed in the proof can be modeled after the one in P. Hartman [2, Example 4.1(6), p. 28] using the techniques in S. G. Deo and G. S. Ladde [1, Theorem 1, p. 47].

\section{REFERENCES}

1. S. G. Deo and G. S. Ladde, Some integral and differential inequalities, Bull. Calcutta Math. Soc. 61 (1969), 47-53. MR 45 \#5513.

2. P. Hartman, Ordinary differential equations, Wiley, New York, 1964. MR 30 \#1270.

3. Z. Mikolajska, Sur l'éxistence d'une solution périodique d'une équation différentielle du premier ordre avec le paramètre retardé, Ann. Polon. Math. 23 (1970/71), 25-36, MR 42 \#627.

4. J. T. Schwartz, Nonlinear functional analysis, Gordon and Breach, New York, 1969.

5. V. V. Strygin, A certain theorem on the existence of periodic solutions of systems of differential equations with retarded arguments, Mat. Zametki 8 (1970), 229-234= Math. Notes 8 (1970), 600-602. MR 43 \#2327.

Department of Mathematics, University of Utah, Salt Lake City, Utah 84112 